\title{
Pour une iconographie de la contestation
}

Towards an iconographic analysis of dissent

\section{Alexandre Dézé}

\section{(2) OpenEdition}

\section{Journals}

\section{Édition électronique}

URL : http://journals.openedition.org/conflits/18773

DOI : $10.4000 /$ conflits. 18773

ISSN : $1777-5345$

Éditeur :

CECLS - Centre d'études sur les conflits - Liberté et sécurité, L'Harmattan

\section{Édition imprimée}

Date de publication : 31 décembre 2013

Pagination : 13-29

ISBN : 978-2-343-02618-3

ISSN : 1157-996X

\section{Référence électronique}

Alexandre Dézé, « Pour une iconographie de la contestation », Cultures \& Conflits [En ligne], 91/92 | automne/hiver 2013, mis en ligne le 31 décembre 2014, consulté le 31 mars 2021. URL : http:// journals.openedition.org/conflits/18773; DOI : https://doi.org/10.4000/conflits.18773 


\title{
Pour une iconographie de la contestation
}

\begin{abstract}
Alexandre DEZE
Alexandre Dézé est Maître de conférences en science politique à l'Université Montpellier 1, chercheur au Centre d'études politiques de l'Europe latine (UMR 5112) et enseignant à Sciences Po Paris. Ses recherches portent actuellement sur la sociologie des organisations partisanes et sur l'iconographie politique. Il a récemment publié : Le Front national : à la conquête du pouvoir ?, Paris, Armand Colin, 2012 ; "Contribution à une approche sociologique de la genèse partisane. Une analyse $d u$ Front national, $d u$ Movimento sociale italiano et $d u$ Front islamique de salut », Revue française de science politique, vol. 61, n 4, 2011 (avec Myriam AïtAoudia).
\end{abstract}

$\mathrm{D}$ es affiches de mai 68 aux murals républicains de Belfast, des caricatures anti-tsaristes de la révolution russe de 1905 au drapeau arc-en-ciel des militants pacifistes, des placards « ouvriers » de Jules Grandjouan au badge "Touche pas à mon pote » de SOS racisme... Quels que soient ses supports ou ses formats, l'image fixe ${ }^{1}$ semble a priori indissociable des pratiques contestataires. Imagine-t-on aujourd'hui une manifestation, une grève ou un sit-in sans banderoles ou pancartes illustrées ? Le symbole graphique du poing levé n'est-il pas la plus simple en même temps que la plus universelle expression de la lutte contre un ordre établi ? Combien d'images - des icônes du Christ détruites au VIII e siècle par les empereurs byzantins ${ }^{2}$ aux T-shirts ornés du triangle rose portés par les premiers militants d'Act-Up à la fin des années $1980^{3}$ - ont-elles participé au déclenchement de mouvements de révolte ou à l'activation de prédispositions à l'engagement ?

1. C'est à ce type d'image que l'on s'intéressera dans cette contribution, laissant ainsi de côté les images dites « animées » ou « mobiles " (film, vidéos, clips) et en « séquences » (bandes dessinées).

2. Voir Besançon A., L'image interdite. Une histoire intellectuelle de l'iconoclasme, Paris, Gallimard, Folio, 2000 [1994].

3. Voir Broqua C., Agir pour ne plus mourir! Act Up, les homosexuels et le sida, Paris, Presses de Sciences Po, 2005. 
L'image semble à ce point avoir intégré la réalité des phénomènes contestataires qu'à force de la voir, on ne la voit plus ${ }^{4}$. On ne peut en effet manquer d'être surpris par le peu d'attention portée à l'iconographie protestataire (ou plus largement aux documents visuels) par les spécialistes de l'action collective. Il existe bien dans la littérature quelques ouvrages et articles de nature historique sur l'affiche sociale ${ }^{5}$, la caricature politique ${ }^{6}$ ou le street art ${ }^{7}$. En revanche, les contributions s'inscrivant dans une perspective de sociologie des mouvements sociaux apparaissent beaucoup plus rares. Pierre Favre s'est certes intéressé à la peinture "sociale » du début du XXe siècle pour rendre compte des modalités de mise en forme des premières manifestations 8 . Jacqueline Adams a analysé les usages militants des broderies tissées au Chili par les femmes des bidonvilles victimes de la répression sous le régime de Pinochet 9 . Thomas Verdon Reed a travaillé sur les fresques murales du mouvement artistique chicano à Los Angeles ainsi que sur le rôle des arts graphiques dans la propagande d'Act Up ${ }^{10}$. Plus récemment, des chercheurs attentifs aux dimensions émotionnelles des mobilisations collectives se sont saisis des images produites par différentes entreprises de protestation pour mieux appréhender leurs « dispositifs de sensibilisation à la cause 11 ». Mais hormis ces quelques exceptions, il faut convenir que l'iconographie ne suscite guère d'intérêt dans le champ de la sociologie des mouvements sociaux : elle n'est quasiment jamais évoquée dans les rencontres scientifiques; elle figure rarement parmi les entrées des dictionnaires et des encyclopédies; elle est traitée de manière marginale dans les publications sur les rapports entre art et contestation; elle peine à être perçue comme un objet permettant de sortir des routines conceptuelles et méthodologiques de la recherche ${ }^{12}$; et finalement, lorsqu'elle est utilisée, c'est le plus souvent avec le «statut dévalorisé de l'illustration ${ }^{13}$ ».

4. Pour paraphraser ici Gisèle Freund lorsqu'elle évoque la place de la photographie dans la vie sociale. Voir Freund G., Photographie et société, Paris, Seuil, 1974, p. 6.

5. Voir par exemple Dumont F., Jouzeau M.-H., Moris J. (eds.), Jules Grandjonan. Créateur de l'affiche politique illustrée en France, Paris, Somogy éditions d'art, 2001 ; Cépède F., Lafon E. (eds.), Le monde ouvrier s'affiche. Un siècle de combat social, Paris, Nouveau Monde Éditions, 2008 ; Weill A. (ed.), Affiches politiques et sociales. Sixièmes rencontres internationales des arts graphiques de Chaumont, Paris, Somogy, 1996.

6. Voir notamment : Dixmier É., Dixmier M., L'Assiette an beurre, Paris, Maspéro, 1974 ; Duprat A., Histoire de France par la caricature, Paris, Larousse, 2000.

7. Chaffee L. G., Political Protest and Street Art. Popular Tools for Democratization in Hispanic Countries, Westport and London, Greenwood Press, 1993.

8. Favre P., «Fixer l'événement. La représentation des manifestations dans la peinture au début du XXe siècle ", in Mény Y. (ed.), Idéologies, partis politiques et groupes sociaux, Paris, Presses de la Fondation nationale des sciences politiques, 1991, pp. 245-263.

9. Adams J., “Art in Social Movements: Shantytown Women's Protest in Pinochet's Chile”, Sociological Forum, 17-1, mars 2002, pp. 21-56.

10. Verdon Reed T., The Art of Protest: Culture and Activism from the Civil Right Movement to the Streets of Seattle, Mineapolis, University of Minesota, 2005, pp. 103-128 et pp. 179-217.

11. Voir Traïni C. (ed.), Émotions... Mobilisation!, Paris, Presses de Sciences Po, 2009.

12. Voir néanmoins Mariette A., " Pour une analyse des films, de leur production à leur réception ", Politix, 93, 2011, pp. 47-68.

13. Selon les termes de Pierre Favre, op.cit., p. 245. Très abondante, l'iconographie remplit précisément cette fonction illustrative dans l'ouvrage de Crettiez X., Sommier I. (eds.), La France Rebelle, Paris, Éditions Michalon, 2006. 
Cet article entend précisément plaider en faveur d'une approche iconographique de la contestation et ambitionne d'en dégager les principaux fondements épistémologiques et méthodologiques. Une telle perspective implique de commencer par interroger les raisons pour lesquelles les documents visuels ont été jusqu'à présent aussi peu étudiés par les spécialistes des mobilisations collectives. Prendre l'image au sérieux nécessite ensuite de poser les conditions de son appréhension comme objet d'étude légitime de la sociologie des mouvements sociaux. Enfin, on tentera de démontrer en quoi l'image peut constituer un matériau de travail heuristique, susceptible d'enrichir notre connaissance des phénomènes contestataires.

\section{Les raisons d'une « myopie conceptuelle»}

L'existence de "points aveugles 14 » dans une discipline ou dans un champ de recherche constitue toujours une curiosité épistémologique. Comment expliquer en l'occurrence que les images, pourtant omniprésentes dans les mobilisations collectives, aient été à ce point négligées par la recherche ? Les raisons d'une telle «myopie conceptuelle 15 » sont évidemment plurielles et tiennent tout à la fois au statut de l'image fixe dans les sciences sociales, au type d'investissement méthodologique que suppose l'analyse iconographique et à la structuration paradigmatique de la sociologie des mouvements sociaux.

Si les images fixes ont été étudiées de manière précoce par les historiens de l'art, elles ont en revanche tardé à être prises en considération comme source documentaire, outil de recherche ou objet d'étude dans les autres disciplines des sciences sociales. En sémiologie, il faut attendre les années 1960 pour que soient posés les premiers fondements d'une rhétorique de l'image. Les travaux de Roland Barthes sont à ce titre décisifs puisqu'ils vont permettre d'établir la nature linguistique de l'image et mettre au jour les mécanismes de production sémantique des messages iconiques, à la jonction entre signifiants (la surface visuelle des signes) et signifiés (leur contenu symbolique, culturel) 16. En anthropologie, l'utilisation de la photographie comme outil de collecte de matériaux va certes accompagner les premiers développements de la discipline à partir du milieu du XIX siècle. Mais elle est progressivement délaissée ${ }^{17}$ et, en définitive, l'anthropologie visuelle ne voit le jour qu'à la fin des années 1960 18. En sociologie, l'intérêt pour les documents iconiques ne s'est vraiment

14. Sur ce point, voir Braud P., L'émotion en politique, Paris, Presses de Sciences Po, 1996, p. 7 et suiv.

15. Selon les termes qu'utilise John. D. H. Downing pour qualifier le désintérêt des spécialistes à l'égard de la communication et des médias comme « dimensions à part entière des mouvements sociaux ». Voir. Downing J. D. H. (ed.), Encyclopedia of Social Movement Media, London, Sage, 2011, pp. xxv.

16. Voir Barthes R., « Rhétorique de l'image », Communications, 4-1, 1964, pp. 40-51.

17. Ce qui n'empêche pas la publication de travaux importants comme ceux de Mead M., Bateson G., Balinese Character, a Photographic Analysis, New York, Académie des sciences de New York, Wilburg, Valentine Editor, 1942.

18. Voir Collier J. Jr, Collier M., Visual Anthropology. Photography as a Research Method, 
manifesté qu'à partir du milieu des années 1970 19. Les réflexions de Howard S. Becker 20 sont parmi celles qui marquent a posteriori les débuts de la « sociologie visuelle » entendue à la fois comme sociologie de l'image et sociologie par l'image. En histoire, la place de l'iconographie est longtemps restée résiduelle avant d'être réévaluée à partir du début des années 1990. Dans un contexte intellectuel de renouvellement des questionnements et des objets de l'histoire politique ${ }^{21}$, des historiens réunis autour du GRIF 22 vont entreprendre de démontrer que l'image peut être utilisée comme preuve et non pas seulement comme illustration ${ }^{23}$. De fait, il n'y a sans doute plus lieu aujourd'hui de parler de « divorce 24 » entre les images et les sciences sociales ${ }^{25}$. En revanche, il faut bien admettre, comme le souligne Christian Delporte, que les études d'iconographie continuent de pâtir d'un véritable "déficit de légitimité scientifique 26 ». Les images apparaissent tout d'abord «marquées du sceau de l'inconséquence et de la frivolité 27 », sans doute parce qu'elles ont été longtemps confinées à un usage essentiellement distractif, que ce soit pour servir d'illustrations à un texte ou remplir des fonctions décoratives. Il faut à ce titre noter le rapport privilégié que les sciences sociales entretiennent avec les sources scripturales. Comme le rappelle Jean-Paul Terrenoire, «les prérogatives de l'écrit se sont faites, et se font encore sentir, à tous les stades de l'analyse et de l'exposition des résultats de la recherche. Finalement, la démarche scientifique dans son entier est marquée en profondeur par ce mode d'expression particulier $28 »$. On peut ensuite convenir que de lourdes présomptions pèsent sur le régime véridictoire des documents visuels et ce au moins depuis l'essor des industries culturelles ${ }^{29}$. Les images étant censées pouvoir faire

Albuquerque, University of New Mexico Press, 1986 [1967].

19. Voir La Rocca F., «Introduction à la sociologie visuelle », Sociétés, 95, janvier 2007, pp. 3340.

20. Voir Becker H. S., "Photography and sociology", Studies in the Anthropology of Visual Communication, 1, 1974, pp. 3-26.

21. Rémond R. (ed.), Pour une histoire politique, Paris, Seuil, 1988.

22. Le "Groupe de recherche sur l’image fixe", constitué entre autres de Fabrice D’Almeida, Laurence Bertrand-Dorléac, Philippe Buton, Christian Delporte, Laurent Gervereau.

23. Pour un bilan de cette entreprise intellectuelle, voir Delporte C., Gervereau L., Maréchal D. (eds.), Quelle est la place des images en histoire ?, Paris, Nouveau monde éditions, 2008.

24. Maresca S., La photographie, un miroir des sciences sociales, Paris, L'Harmattan, 1996, p. 121.

25. Cette sensibilité aux images comme objet d'étude ou matériau de travail est même désormais repérable dans des champs sous-disciplinaires comme les relations internationales. Voir par exemple les analyses d'Anni Kangas sur les dessins politiques finlandais du début du XXe siècle, ou de Klaus Dodds sur les représentations géopolitiques dans les dessins de Steve Bell pour le journal britannique The Guardian: Kangas A., "From Interfaces to Interpretants: A Pragmatist Exploration into Popular Culture as International Relations", Millennium: Journal of International Studies, 38-2, 2009, pp. 317-343 ; Dodds K., "Steve Bell's Eye: Cartoons, Geopolitics and the Visualization of the 'War on Terror', Security Dialogue, vol. 38-2, 2007, pp. 157-177. Je remercie l'un des deux évaluateurs anonymes de cet article pour m'avoir signalé ces contributions.

26. Delporte C., « De la légitimation à l'affirmation », in Delporte C., Gervereau L., Maréchal D. (eds.), op.cit., p. 11.

27. Terrenoire J.-P., «Images et sciences sociales : l'objet et l'outil », Revue française de sociologie, 26-3, 1985, p. 509.

28. Ibid, p. 511.

29. De Gournay C., «Le deuil de l'image. De la photographie à l'image virtuelle », Réseaux, 61, 1993, p. 127. 
l'objet de toutes les manipulations, et, partant, ne jamais dire le vrai, elles apparaissent toujours moins fiables comme «preuves » que d'autres types de sources (pourtant tout aussi manipulables ${ }^{30}$ ). Il importe enfin de se rappeler que le processus de constitution des sciences sociales (tout du moins à partir du début du XX $\mathrm{XX}^{\mathrm{e}}$ siècle ${ }^{31}$ ) s'est accompagné d'un rejet de « tout ce qui paraissait difficilement mesurable par des méthodes empiriques de recherche 32 ». Or les propriétés polysémiques du discours iconique ${ }^{33}$, qui semblent ouvrir sur une palette infinie de possibles interprétatifs, tendent précisément à lui conférer un caractère a priori insaisissable. En somme, l'image reste avant tout perçue comme un matériau "non scientifique 34 » en sciences sociales notamment en sociologie, où la recherche iconographique semble avoir plus de difficultés à se développer que dans d'autres disciplines.

Si les documents iconographiques ont été à ce point négligés par la recherche sur les mouvements sociaux, c'est aussi, peut-être, parce que leur analyse suppose l'intervention d'un dispositif méthodologique de type interdisciplinaire. Certes, le plus souvent, l'analyse iconographique s'effectue à partir d'un point de vue monodisciplinaire (historique, sociologique, anthropologique, etc.). Cela dit, la sémiologie fournit de précieux outils pour déconstruire les processus de sémantisation du discours graphique ; la sociologie de la réception s'avère indispensable pour comprendre les modalités d'appropriation de ce discours par les agents sociaux; l'histoire est tout autant incontournable pour saisir non seulement les caractéristiques contextuelles de production de supports iconographiques mais également les références culturelles qu'ils mobilisent. D'un point de vue méthodologique, l'adoption d'une démarche interdisciplinaire apparaît donc toujours souhaitable devant l'image. Il reste qu'une telle démarche est souvent associée à une sorte de braconnage intellectuel illicite et qu'elle s'avère souvent rebutante car coûteuse en temps (elle «n'est pas de tout repos ", écrivait Roland Barthes ${ }^{35}$ ). Surtout, comme le relève Isabelle Sommier, « le défi de l'interdisciplinarité » semble « d'autant plus difficile à relever » dans le domaine d'étude des mouvements sociaux que la construction de ce domaine « s'est accompagnée d'un repli sur soi et d'une ultraspécialisation 36 ». En somme, une telle perspective méthodologique apparaît peu conciliable avec les orientations de la recherche.

30. Sur cette question centrale du régime véridictoire des images, voir Becker H. S., « Les photographies disent-elles la vérité ?", Ethnologie française, 37-1, 2007, pp. 33-42.

31. Comme le rappelle Sylvain Maresca, l'image (pour l'essentiel la photographie) a bien accompagné les premiers développements des sciences sociales, dans la deuxième moitié du XIXe siècle, avant de faire l'objet d'une critique scientiste de plus en plus marquée et d'une disqualification comme matériau. Voir Maresca S., op.cit., p. 122 et suiv.

32. Sommier I., "Les états affectifs ou la dimension affectuelle des mouvements sociaux », in Fillieule O., Agrikoliansky, Sommier I. (eds.), Penser les mouvements sociaux, Paris, La Découverte, 2010, p. 189.

33. Voir Barthes R., L'obvie et l'obtus. Essais critiques III, Paris, Seuil, 1982, pp. 9-24.

34. Voir Becker H. S., "Les photographies...», op.cit.

35. Barthes R., Le bruissement de la langue. Essais critiques IV, Paris, Seuil, 1984, p. 69.

36. Sommier I., "Les états affectifs ...», op.cit., p. 189. 
La façon dont s'est construite cette recherche, les pratiques scientifiques qui s'y sont développées, permettent de comprendre en dernier ressort pourquoi l'image y est tenue à l'écart. Comme on le sait, la sociologie des mouvements sociaux s'est bâtie sur un rejet des théories psychologisantes - dominantes depuis les théories des foules du XIXe siècle jusqu'aux analyses en termes de frustration des années 1960 - au profit d'une conception de plus en plus étroitement utilitariste de l'action collective. Guidés par les modèles de la mobilisation des ressources et du processus politique, les travaux se sont ainsi longtemps détournés de la dimension subjective des phénomènes contestataires (rendant par conséquent improbable toute prise en compte des matériaux iconographiques). Le développement de l'analyse des cadres marque a posteriori une première rupture avec ces orientations de recherche et préfigure l'avènement d'un courant qualifié de « culturel 37 ». En reposant la question de la fabrique du sens dans l'action collective, certains sociologues vont être amenés à élargir leur champ d'observation et à étudier les pratiques contestataires au prisme de leur dimension idéelle, morale, affectuelle, identitaire. L'image constitue a priori un support d'accès privilégié à cette sphère sensible des phénomènes sociaux. Le développement d'une sociologie culturelle de l'action collective aurait donc dû favoriser son intégration comme matériau de travail. Pourtant, l'image continue d'occuper une place mineure dans la recherche. Ce désintérêt persistant témoigne finalement assez bien d'une certaine routinisation des pratiques d'analyse ${ }^{38}$. La plupart des contributions portant sur les rapports entre art et contestation en offrent à ce titre une bonne illustration, puisqu'elles prolongent généralement les questionnements ordinaires de la recherche (analyse des trajectoires individuelles, des logiques d'engagement, des dynamiques organisationnelles ${ }^{39}$ ) et ne conçoivent que rarement la possibilité de se saisir des productions artistiques en tant que telles (exception faite de la « musique engagée $40 »$ ) pour étudier les mouvements de contestation.

\section{Construire l'image comme objet d'étude sociologique des phénomènes contestataires}

On comprend sans doute un peu mieux pourquoi l'image fixe est restée à ce point absente des travaux sur l'action collective. Pourtant, il est manifeste que la recherche a tout à gagner à se saisir de la réalité des phénomènes contestataires «dans ce qu'elle a et par ce qu'elle a de visible 41 ». Ce qui implique au

37. Sur « l'invention » de cette « sociologie culturelle » des mouvements sociaux, voir Cefaï D., Pourquoi se mobilise-t-on? Les théories de l'action collective, Paris, La Découverte, 2007, pp. 467-545.

38. Sur ce point, voir Combes H. et al. « Observer les mobilisations. Retour sur les ficelles du métier de sociologue des mouvements sociaux », Politix, 24-93, 2011, p. 14.

39. Voir par exemple Balasinski J., Mathieu L. (eds.), Art et contestation, Rennes, Presses Universitaires de Rennes, 2006 ; Roussel V., Arts vs war. Les artistes américains contre la guerre en Irak, Paris, Presses de Sciences Po, 2011.

40. Voir notamment Traïni C., La musique en colère, Paris, Presses de Sciences Po, 2008.

41. Terrenoire J.-P., op.cit., p. 512. 
préalable de construire l'image comme objet d'étude légitime de ces phénomènes.

Une telle perspective nécessite tout d'abord d'en préciser les contours définitionnels. Par image fixe, on entendra ici tout «manifeste » qui comporte une représentation de quelque chose, accompagné ou non d'éléments textuels, et qui peut s'inscrire sur différents supports ${ }^{42}$. Deux types de "manifeste » sont susceptibles d'intéresser les spécialistes de l'action collective : les supports représentant des phénomènes contestataires (photographies, dessins de presse, cartes postales, tableaux de peinture, etc.) et les supports iconiques fabriqués ou utilisés à des fins de protestation par des acteurs collectifs porteurs d'une cause (affiches, pancartes, banderoles, badges, flyers, etc.). Il faut préciser ici qu'il n'existe pas d'image contestataire en soi. Une image peut ne pas être contestataire et le devenir. Lorsqu'à l'occasion d'une marche organisée contre la guerre du Vietnam en 1967, Marc Riboud photographie Jan Rose Kasmir, cette jeune femme qui tient une fleur entre ses mains à quelques centimètres des baïonnettes des fusils des soldats de la garde nationale américaine, il ne produit rien d'autre sur l'instant qu'un cliché destiné à être vendu par son agence de presse. En revanche, lorsque ce même cliché est brandi sur une pancarte en 2003, à Londres, par des manifestants hostiles à la guerre en Irak (parmi lesquels Jan Rose Kasmir) il revêt bien - de par l'utilisation spécifique qui en est faite - une signification protestataire (cf. document 1$)$.

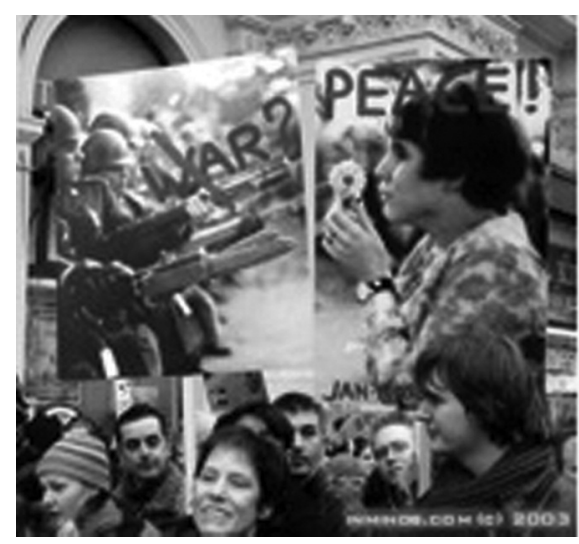

Document 1 - Manifestation organisée en 2003 à Londres contre la guerre en Irak. Jane Rose Kasmir (au centre en bas) défile en-dessous d'une pancarte sur laquelle est reproduite la photo prise par Marc Riboud en 1967

42. On s'inspire ici directement de la définition de Jean-Paul Gourévitch in Gourévitch J.-P., L'image en politique. De Luther à Internet, Paris, Hachette Littératures, 1998, p. 11. 
Il faudrait bien sûr s'attacher à reconstituer le processus complexe par lequel une simple photographie, aussi réussie soit-elle, en vient à être « dépassée " par ce qu'elle représente pour s'imposer comme un "symbole de condensation 43 » de la cause pacifiste. Mais en l'occurrence, on aura compris que le sens protestataire qui peut être assigné aux images dépend tout à la fois des usages qui en sont faits, du contexte de leur utilisation et des schèmes culturels des récepteurs. Ainsi, la figure iconique du « Che 44 » ne revêt pas la même signification lorsqu'elle est utilisée à La Havane dans le cadre d'un dispositif d'inscription des «normes révolutionnaires » dans le paysage urbain ${ }^{45}$, lorsqu'elle sert d'illustration à des paquets de cigarettes ou à des bouteilles de rhum vendues dans le commerce occidental, ou lorsqu'elle orne les tentures de boutiques de luxe parisiennes ( $c f$. documents 2-1 à 2-3). Le sens d'une image n'est jamais déterminé a priori mais fait l'objet d'investissements politiques et symboliques qui en garantissent la portée.
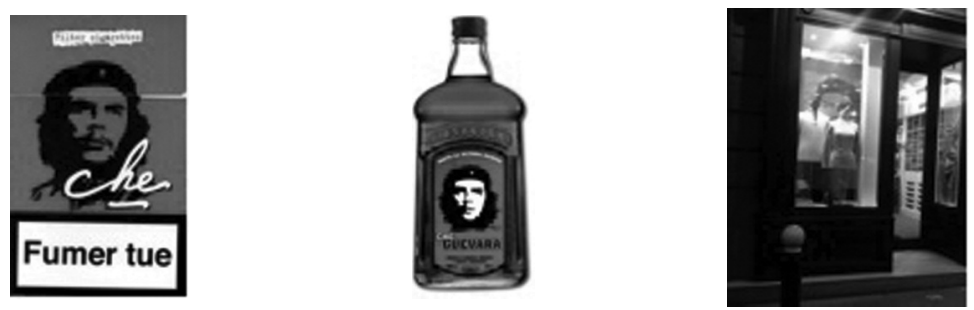

Documents 2-1, 2-2, 2-3 - La figure du «Che » utilisée pour le merchandising de produits de consommation et la décoration de boutiques parisiennes

Construire l'image comme objet d'étude implique ensuite de préciser les conditions de légitimité de son utilisation dans le cadre d'une approche sociologique. Si l'image est susceptible d'intéresser les sociologues, c'est parce qu'elle ne constitue pas seulement un système de signes (comme la conçoivent les sémiologues), une structure morphologique de perception (comme la conçoivent les psychologues) ou une source documentaire (comme la conçoivent les historiens). L'image procède en effet plus largement d'un acte de communication qui est tout à la fois le produit de pratiques sociales et qui met en jeu des rapports sociaux ${ }^{46}$. Cette dimension proprement sociale de la construction des images est le plus souvent évacuée dans les analyses iconographiques, qui se concentrent généralement sur l'examen de leur matérialité

43. Sapir E., Anthropologie, Paris, Seuil, 1971.

44. Figure issue du portrait photographique réalisé par Alberto Korda en 1960 et popularisée en 1968 par l'affiche rouge et noir de Jim Fitzpatrick.

45. Geoffray M.-L., Contester à Cuba, Paris, Dalloz, 2012, p. 135.

46. Sur l'art comme produit d'une « activité collective », voir Becker H. S., Les mondes de l'art, Paris, Flammarion, 1988. 
interne. On s'attache ainsi rarement à l'étude des modalités de production des documents visuels, de leurs usages, des interactions qu'ils engagent ou encore de leur interprétation par le public. On ne saurait cependant oublier que l'image est « immergée » dans le social et qu'elle « ne livre finalement son sens que dans le discours et les actes de l'individu ou du groupe qui l'objective en le disant ou en en faisant quelque chose 47 ». Or quelle discipline, mieux que la sociologie, est susceptible d'offrir les outils adaptés à la saisie de l'émission et de la réception d'une image et des processus complexes qui les relient 48 ? La sociologie apparait ainsi aussi légitime que n'importe quelle autre discipline pour intervenir dans l'enquête iconographique; elle s'avère même parfaitement complémentaire aux approches internes. Si le «principe de pertinence 49 » de la sémiologie réside dans l'analyse immanente du sens de l'image, celui de la sociologie se situe dans l'analyse de la façon dont ce sens se construit dans l'interaction entre l'émetteur et ses récepteurs ${ }^{50}$. Délimiter de la sorte le champ d'intervention de la sociologie ne signifie pas pour autant qu'il faille s'interdire de s'intéresser au contenu des images. Comme le rappelle Jean-Paul Terrenoire, l'image se donne aussi comme « une matrice de rapport social » où émetteur, message et récepteur sont toujours " co-présents 51 ». De fait, la façon dont l'émetteur se représente dans l'image, la manière dont il construit en creux la figure du destinateur, la vision du monde qu'il projette ou les valeurs qu'il mobilise intéressent aussi directement la sociologie.

Prendre l'image au sérieux comme objet d'étude nécessite enfin de s'en remettre à des procédures d'analyse normalisées. La lecture d'une image ne requiert a priori aucune compétence particulière, ce qui peut sembler suffisant pour justifier une forme d'exceptionnalisme méthodologique. Il reste que la normalisation de l'objet passe nécessairement par la normalisation de son approche. Une telle perspective implique tout d'abord d'appréhender l'image non plus comme une simple illustration mais comme un matériau premier. Il s'agit ainsi, comme le rappelle Marc Ferro, de "partir de l'image, des images », de «ne pas chercher seulement en elles illustration, confirmation, ou démenti à un autre savoir, celui de la tradition écrite ", mais de les «considérer [...] telles quelles 52 », au même titre donc qu'une série statistique, un entretien ou une archive. L'analyse de l'image (et non plus sa simple lecture) engage ensuite des savoir-faire spécifiques. La plupart des spécialistes de l’image s'accordent

47. Passeron J.-C., Le raisonnement sociologique. L'espace non-poppérien du raisonnement naturel, Paris, Nathan, 1991, p. 269.

48. Ce que rappelle notamment R.Barthes, dans: L'obvie et l'obtus, op.cit., p. 9.

49. Barthes R., "Éléments de sémiologie », Communications, 4, 1964, p. 132.

50. Pour une illustration appliquée au cas de l'affiche politique, voir Dézé A., «L'image en questions. Retour sur une enquête de réception du discours graphique du Front national ", in Favre P., Fillieule O., Jobard F. (eds.), L'Atelier du politiste. Mélanges Pierre Favre, Paris, La Découverte, 2007, pp. 313-330.

51. Jean-Paul Terrenoire, op. cit., p. 514.

52. Ferro M., "Le film, une contre-analyse de la société ", Annales. Économies, Sociétés, Civilisations, 28-1, 1973, p. 113. 
sur une démarche héritée de l'iconologie 53 et de la sémiologie ${ }^{54}$ qui procède d'une première étape descriptive du message de dénotation de l'image suivie d'une deuxième étape interprétative de son message de connotation. Pour simplifier, on peut dire que le message de dénotation désigne la partie proprement visible de l'image. Dans l'affiche $d u$ «Che », par exemple, ce message se compose d'un visage dessiné à l'encre noir sur un fond de couleur rouge (ce que tout un chacun est capable de percevoir). Le message de connotation constitue en quelque sorte l'extension du message de dénotation. S'il ne s'inscrit pas dans la matérialité propre de l'image, il se laisse en revanche deviner « derrière » sa partie visible : il est le dépositaire de la signification de l'image, signification qui dépend cependant des ressources interprétatives des récepteurs et de leurs schèmes culturels. Dans l'affiche du « Che », le dessin de couleur noir sur fond rouge pourra ainsi potentiellement et variablement « signifier » le visage d'un individu quelconque, une figure majeure de l'histoire cubaine, un symbole de l'anti-américanisme, un appel à la mobilisation sociale, etc. Cette démarche méthodologique en deux temps constitue en général l'armature de base des grilles d'analyse mobilisées dans les enquêtes iconographiques, grilles qui peuvent revêtir des formes plus ou moins sophistiquées. Dans le modèle qui est proposé ici (voir infra), il s'agit au niveau descriptif de repérer les constituants morphologiques de l'image, d'opérer un relevé des différents éléments textuels et iconiques, d'interroger les rapports fonctionnels entre texte et image, et, si l'on veut aller un peu plus loin, de procéder à une analyse « actantielle 55 » du discours graphique et de son programme narratif. La deuxième étape interprétative est réputée pour être plus délicate. D’aucuns évoquent en effet, face à l'image, une « crise de l'interprétation 56 » qui serait

53. Panofsky E., Essai d'iconologie. Les thèmes humanistes dans l'art de la Renaissance, Paris, Gallimard, 1967 [1939].

54. Voir par exemple Joly M., L'image et les signes. Approche sémiologique de l'image fixe, Paris, Nathan, 1994.

55. L'approche actantielle a été mise au point par le sémioticien Algirdas Julien Greimas à partir des travaux de Vladimir Propp sur la morphologie des contes populaires. Appliquée à l'analyse de l'image, cette approche vise à repérer dans la matérialité iconique les différentes instances participant de la construction de sa narration et leur fonction syntaxique (voir Memmi D., Du récit en politique. L'affiche électorale italienne, Paris, Presses de la FNSP, 1986). Les actants fonctionnent par paires selon un schéma actantiel (sujet-objet, destinateur-destinataire, adjuvant-anti-sujet). Dans le cadre d'une sociologie par l'image des mouvements sociaux, cette grille peut fournir un outillage intéressant pour étudier la structure minimale du discours iconique protestataire (un discours qui est le plus souvent conflictuel), et notamment la façon dont s'ordonnent, dans les différents supports d'un même corpus, les rapports entre le sujet (l'entrepreneur de cause, les militants, le peuple...), l'anti-sujet (autrement dit l'adversaire qui est combattu) et l'objet de valeur (ce qui est revendiqué, promu ou défendu). C'est notamment sur ces trois figures actantielles que se concentre l'analyse que propose Philippe Buton de l'iconographie communiste française et italienne après 1945. L'article ne porte donc pas spécifiquement sur les mobilisations collectives, mais l'approche qu'il propose, inspirée des travaux de Dominique Memmi, apparait parfaitement transposable. Il s'agit alors de déterminer sous quelles formes et dans quelle proportion (en termes quantitatifs) l'ennemi est représenté dans les affiches politiques, comment s'incarne variablement le sujet (tantôt le peuple, le parti ou le dirigeant), et quels quête ou objet de valeur poursuit le communisme (horizons d'attente, objectifs, espoirs). Voir Buton Ph., "L'adieu aux armes ? L'iconographie communiste française et italienne après la Libération ", Vingtième Siècle, 80, 2004, pp. 43-54. 
liée à son caractère immanquablement polysémique. Il s'agit là cependant d'un phénomène qui est loin d'être spécifique à l'analyse iconographique et qui se pose plus largement dans le cadre de toute démarche interprétative. L'interprétation de la signification d'une image est à rechercher dans les «systèmes d'associations » (c'est-à-dire les « codes ») mis en jeu qui permettent d'assurer le passage du niveau dénotatif au niveau connotatif du discours graphique. Chaque code puise dans un système de conventions culturellement établies. Prenons l'exemple des codes chromatiques : le rouge (dénotatif) peut être utilisé pour signifier l'amour ou la révolte (connotatif). Le blanc (dénotatif) peut être utilisé pour signifier, au niveau connotatif, la pureté (dans un contexte occidental) ou le deuil (en Chine, par exemple). Chaque code fonctionne ainsi comme un producteur de signification. Si l'on prend l'exemple des codes symboliques, le poing levé (dénotatif) est généralement utilisé pour signifier la révolte (connotatif), la chaîne (dénotatif) pour signifier l'oppression (connotatif). L'analyse interprétative consiste précisément à repérer ces différents codes - au niveau iconique, symbolique, rhétorique, typographique, chromatique, axiologique, etc. - à les déconstruire, et à émettre des hypothèses sur les diverses significations possibles de l'image - hypothèses qui peuvent être contrôlées par une enquête auprès de l'émetteur et des récepteurs. Cette étape interprétative se conclut enfin par une interrogation sur les fonctions (informative, expressive, incitative, phatique) de l'image inspirées des travaux de Roman Jakobson ${ }^{57}$. Il est certes tout à fait possible de se dispenser de ce type d'outil pour analyser des documents visuels. Cependant, dans la mesure où l'utilisation d'une telle grille garantit un travail d'objectivation systématique de l'image, elle peut s'avérer précieuse pour réduire les risques de biais interprétatifs et permettre un comparatisme plus systématisé. Dans une même logique de normalisation méthodologique, rappelons que l'on gagne également à débuter toute enquête iconographique par un travail préalable de lecture du corpus et de renseignement des documents visuels qui le constituent (voir la rubrique « Préalables » de la grille).

56. Louvet J.-R., « Du proche au lointain : les images en histoire », in Coll., Image et histoire. Actes du colloque Paris-Censier. Mai 1986, Paris, Publisud, 1986, p. 26.

57. Jakobson R., Essais de linguistique générale, Paris, Minuit, 1963. 


\section{Grille d'analyse des images fixes}

\begin{tabular}{|c|c|}
\hline \multicolumn{2}{|l|}{ 1) PRÉALABLES } \\
\hline Lecture des images & $\begin{array}{l}\text { Prendre le temps de se familiariser avec le corpus en arrêtant son regard sur } \\
\text { les images. Retranscrire éventuellement ses impressions premières. }\end{array}$ \\
\hline Fiche technique & $\begin{array}{l}\text { Renseigner toutes les informations disponibles sur les images constitutives du } \\
\text { corpus } \\
\text { - Titre (éventuel) } \\
\text { - Type de document (affiche, photo, dessin, peinture, sticker, T-shirt...) } \\
\text { - Technique de conception (gravure, lithographie, etc.) } \\
\text { - Source } \\
\text { - Format, Tirage } \\
\text { - Émetteur / Auteur / Concepteur / Commanditaire } \\
\text { - Éditeur / Imprimeur } \\
\text { - Date d'émission, contexte de production et de réception (impact, notoriété, } \\
\text { reprise, détournement) }\end{array}$ \\
\hline
\end{tabular}

2) DESCRIPTION

\begin{tabular}{|l|l|}
\hline $\begin{array}{l}\text { Approche dénotative } \\
\text { (plan d'expression) }\end{array}$ & Approche descriptive des signifiants de l'image \\
& - Description linéaire et globale de l'image (Que voit-on ?) \\
- Inventaire des signes morphologiques (composition, lisibilité, zones de \\
préemption visuelle, répartition des zones textuelles et iconiques, lignes de \\
force, points d'ancrage, sens de lecture, cadrage, perspective, etc.) \\
- Inventaire des signes iconiques (ensemble des éléments visuels représen- \\
tés : lieux, décors, symboles, personnages, attributs, couleurs, etc.) \\
- Inventaire des signes textuels (titre, slogan, légende, mode d'identification, \\
mode d'interpellation, bandeau, bulles, incrustations, etc.) \\
- Rapport fonctionnel entre le texte et l'image (ancrage, relais, redondance, \\
contradiction, etc.) \\
- Repérage des modalités de construction et de distribution du jeu des \\
actants (destinateur, destinataire, objet de valeur, sujet, anti-sujet et adju- \\
vant) et de la structure du programme narratif
\end{tabular}

\section{3) INTERPRETATION}

\begin{tabular}{|l|l|}
\hline $\begin{array}{l}\text { Approche connotative } \\
\text { (plan de contenu) }\end{array}$ & $\begin{array}{l}\text { Approche interprétative des signifiés de l'image } \\
\text { - Évaluation des systèmes d'association mis en jeu : codes morphologique, } \\
\text { iconique, rhétorique, graphique, typographique, chromatique, symbolique, } \\
\text { axiologique, etc. } \\
\text { - Repérage des fonctions de l'image (plus particulièrement les fonctions } \\
\text { informative, expressive, incitative et phatique) } \\
\text { - Analyse (éventuelle) de réception auprès d'un échantillon (saisie in situ, } \\
\text { entretiens projectifs, protocoles expérimentaux) et enquête (éventuelle) } \\
\text { auprès de l'émetteur }\end{array}$ \\
\hline
\end{tabular}

\section{Étudier les mouvements sociaux par l'image fixe}

Si l'image fixe peut être construite comme objet d'étude légitime des phénomènes contestataires, on souhaiterait ici brièvement démontrer en quoi une approche iconographique des mouvements sociaux est susceptible de permettre de dessiner des pistes de recherche potentiellement génératrices de nouveaux gains de connaissance. Trois pistes (parmi d'autres) peuvent être évoquées ici. 
Une première piste consiste à se saisir de l'image comme parole protestataire. Comme le souligne Lilian Mathieu, « les mouvements sociaux sont des gros producteurs de discours ", et cette " production discursive », qui peut représenter dans certains cas "l'essentiel de leur activité », " constitue un matériau de premier choix pour l'analyse ». L'auteur évoque alors à titre d'exemple les «programmes de réforme », les «tracts », la «presse militante », ou la « prise de parole lors de réunions publiques 58 ». Il omet cependant de mentionner les images. Or ce type de support permet comme les autres, voire peut-être mieux que les autres, d'investir le discours protestataire des mobilisations collectives. Il suffit d'observer une manifestation : c'est notamment par l'image - pancartes, banderoles, ballons, drapeaux, bannières, stickers, badges illustrés - que s'exprime le corps manifestant, qu'il donne à voir (et non pas seulement à entendre) sa vision du monde, ses valeurs, ses revendications ${ }^{59}$. De fait, si l'on veut comprendre le sens qui est investi dans la contestation, il importe de s'attacher à l'étude de ces dispositifs d'énonciation iconiques. L'analyse de leur contenu semble dès lors assez bien adaptée pour rendre compte du travail de cadrage opéré par les entrepreneurs de cause ${ }^{60}$. Les représentations visuelles constituent a priori des supports heuristiques pour saisir les modalités de construction sémantique d'une réalité sociale donnée. Les photos, les dessins qui sont érigés lors des mobilisations participent pleinement des luttes de sens qui s'y engagent. Et si ces images y sont si abondantes, c'est peut-être parce qu'elles fonctionnent comme autant de raccourcis cognitifs, tendant ainsi à faciliter ce travail «d'assignation d'une signification » à l'engagement pour une cause ( $c f$. documents 3-1 à 3-3). De la même manière, l'examen de la production (c'est-à-dire du choix des supports, des thématiques, des visuels, des éléments de langage textuel, etc.) et de la diffusion (collage, tractage, e-mailing, etc.) de ces dispositifs iconiques pourrait fournir d'utiles renseignements sur les pratiques militantes intervenant en amont de la production du fait contestataire. On gagnerait à ce titre à s'intéresser davantage aux pratiques d'affichage, qui constituent tout à la fois un moment souvent important de la sociabilité militante, une opération incontournable du travail de mobilisation et un moyen par lequel s'inscrit la contestation dans l'espace social. Enfin, on peut supposer, dans une même perspective, que l'étude des modalités d'appropriation des visuels contestataires (que ce soit en contexte ou a posteriori, au moyen d'entretiens projectifs ${ }^{61}$ ) serait à même

58. Mathieu, L. L'espace des monvements socianx, Bellecombes-en-Bauges, Éditions du Croquant, 2012, p. 253.

59. Pour une analyse de la contribution des corps à la définition du phénomène manifestant, voir Soutrenon E., "Le corps manifestant. La manifestation entre expression et représentation ", Sociétés contemporaines, 31, 1998, pp. 37-58.

60. Pour une approche iconographique de ce travail de cadrage, voir Adams J., op.cit.

61. On a pu proposer ce type de dispositif dans le cadre d'une enquête de réception des affiches du Front national. Il s'agissait alors de comprendre la façon dont le discours graphique frontiste pouvait être investi, compris et interprété à l'aune de l'intention émettrice (saisie par entretien), du contenu du message (appréhendé par une analyse interne) et du contexte de réception (à partir d'une observation de terrain). Trois protocoles ont été mis en œuvre : une enquête par questionnaires réalisée dans plusieurs villes devant les panneaux d'affichage des 
d'offrir de précieuses indications sur la signification que les agents pensent conférer à leur action.
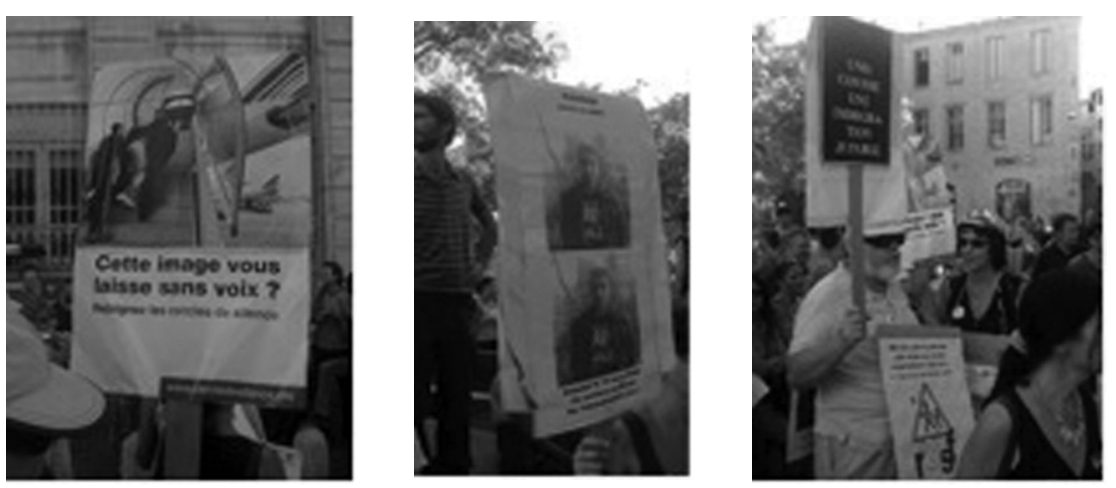

Documents 3-1, 3-2, 3-3 - Manifestation de collectifs de défense des sans-papiers, Montpellier, septembre 2010

Une deuxième piste consiste à partir des images pour analyser les modalités de production des identités collectives des mouvements contestataires. Les entrées sont ici multiples. L'image peut tout d'abord être abordée comme une «technologie » permettant à des groupements d'individus de se faire connâ̂tre et reconnaître en tant que collectifs protestataires. Comme le rappellent Olivier Fillieule et Danielle Tartakowsky à propos de la manifestation, « la construction d'identités collectives stratégiques s'appuie sur les scénographies manifestantes. Ces scénographies renvoient d'abord à une multiplicité de signes de reconnaissance et d'identification comme les bannières, drapeaux, banderoles, badges [...] ${ }^{62}$. L'utilisation de ce matériel est essentiel à la production du fait manifestant, mais il joue également un rôle d'ordonnancement identitaire au sein même des cortèges : les manifestants ne défilent pas derrière n'importe quelle bannière ou sous n'importe quel drapeau. Ils s'assemblent autour de dispositifs iconiques différenciés qui contribuent à leur identification (les rassemblements du $1^{\text {er }}$ mai en France en offrent une illustration) ou à l'image qu'ils souhaitent donner d'eux-mêmes et de leur mouvement - autant d'aspects qui mériteraient sans doute d'être davantage pris en compte par la recherche. L'image participe plus largement des procédés de construction des communautés contestataires. Les symboles, notamment, font partie de ces signes distinctifs « qui vont conduire les membres [d'un groupe] à se recon-

élections régionales de 1998 ; des entretiens collectifs basés sur la projection d'affiches du FN (truquées par ordinateur) visant à cerner les modalités de reconstruction du discours frontiste et à en dégager ainsi les propriétés singulières; des entretiens projectifs individuels de type non directif à partir d'un échantillon d'affiches du FN. Pour une présentation plus détaillée de ces protocoles et des résultats, voir Dézé A., "L’image en questions. ...», op.cit.

62. Fillieule O., Tartakowsky D., La manifestation, Paris, Presses de Sciences Po, 2008, p. 155. 
naître à la fois comme différents de ceux qui sont hors du groupe (identisation) et comme semblables à ceux qui sont dans le groupe (identification) 63 ». Ils contribuent donc à l'édification des frontières identitaires des collectifs contestataires en dessinant un ensemble de principes et de références partagés ; mais ils constituent également de puissants moyens d'attachement des individus à ces collectifs. Christophe Broqua souligne à ce titre l'importance des usages du symbole du triangle rose dans le travail entrepris par les responsables de l'association Act Up pour constituer une " "communauté homosexuelle" latente en groupe organisé 64 ». Il démontre notamment comment le port du T-shirt sur lequel est reproduit ce symbole, aux résonnances politiques dramatiques, s'impose progressivement comme un mode d'expression d'appartenance à cette communauté mais aussi comme une marque d'engagement dans la lutte contre le SIDA 65 ( $c f$. document 4). On peut cependant s'étonner du peu d'intérêt qui est généralement porté à la production symbolique des mouvements contestataires. On ne sait finalement pas grand-chose de ces supports iconiques de la lutte sociale qui s'imposent souvent à nous avec la force de l'évidence. L'analyse de leur genèse et de leur fabrique serait pourtant susceptible de nous renseigner sur le travail de définition originel de la cause que les entrepreneurs entendent défendre. De même, l'examen de leurs usages par les militants pourrait permettre de mieux comprendre leur rapport aux revendications contestataires et plus largement au mouvement qui les porte. Enfin, l'observation de leur circulation dans l'espace des mouvements sociaux serait à même de nous informer sur les phénomènes de transnationalisation des pratiques contestataires. L'image peut encore être sollicitée pour étudier les dispositifs stratégiques d'attention médiatique déployés par les mouvements sociaux (dispositifs qui contribuent pleinement à leur construction identitaire). Il n'est pas rare que les caméras ou les appareils-photos des journalistes se focalisent sur les éléments visuels des actions collectives, notamment parce qu'ils participent de leur spectacularisation. Cette focalisation peut conduire les acteurs à prêter, par anticipation, un soin tout particulier à leur élaboration, non seulement pour s'assurer de la bonne diffusion de leurs revendications mais également pour garantir une représentation valorisante du groupe mobilisé. Le corps devenu image des militantes du mouvement Femen en offre à ce titre une assez bonne illustration ( $c f$. document 5 ). De la même manière, les ressources iconographiques peuvent s'avérer propices pour examiner les « dispositifs de sensibilisation mémorielle 66 » des mouvements sociaux. Ces dispositifs ont notamment pour finalité d'entretenir les fondements identitaires des communautés militantes en générant des réactions affectives partagées : c'est le cas, par exemple, des murales peints par

63. Chevallier J., «Identité, organisation, institution », in CURAPP, L'identité politique, Paris, Presses universitaires de France, 1994, p. 239.

64. Broqua C., Agir..., op.cit., p. 129.

65. Ibid, p. 399.

66. Traïni C., Siméant J., " Pourquoi et comment sensibiliser à la cause ? », in Traïni C. (ed.), Émotions..., op.cit., p. 25. 
les artistes « chicanos » engagés dans la lutte pour la reconnaissance de la communauté mexicaine aux États-Unis et qui offrent nombre de représentations commémoratives des figures et des événements de l'histoire oubliée de cette communauté ${ }^{67}$; c'est également le cas des portraits photographiques des militants d'Act Up décédés, reproduits sur des pancartes et brandis par les membres de l'association sur la place de la Concorde en 1993. Or il faut bien reconnaître que ces différents aspects restent à ce jour peu pris en compte par la recherche.

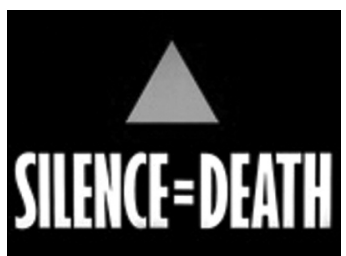

Document 4 - le logo d'Act Up

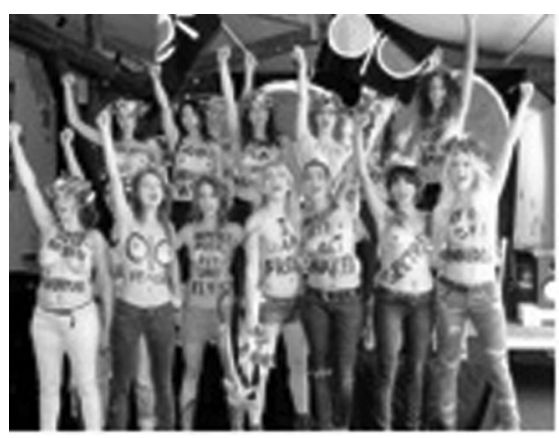

Document 5 - Les militantes du mouvement FEMEN

Une troisième piste consiste à s'intéresser à l'image pour mieux comprendre les logiques complexes de passage à l'acte. Cette voie semble avoir été davantage explorée, notamment depuis les travaux de James M. Jasper et Jane D. Poulsen sur les « chocs moraux ». Ainsi, les organisations, les réseaux ne constitueraient pas les seuls moyens de recruter des individus. L'utilisation de supports matériels capables de susciter des réactions indignées favoriserait également l'engagement pour une cause. Évoquant les mouvements de défense des droits des animaux, James M. Jasper et Jane D. Poulsen soulignent notamment les effets mobilisateurs liés aux usages militants de photos de chiens affamés, de taureaux ensanglantés ou de singes enfermés ${ }^{68}$. Dans une veine similaire, Christophe Traïni démontre comment l'essentiel du travail militant déployé par certaines associations anti-corrida consiste à provoquer la sensibilité d'un public initialement indifférent en usant d'images qui visent à « frapper les sens » et à «toucher les cœurs 69 ». L'iconographie est de la même manière considérée comme un matériau de première main par Axelle Brodiez qui cherche à mettre au jour les stratégies d'indignation utilisées dans la communication des organisations humanitaires ${ }^{70}$. Aucun de ces travaux n'ignore

67. Verdon Reed Th., op.cit.

68. Jasper J. M., Poulsen J. D., "Recruiting Strangers and Friends: Moral Shocks and Social Networks in Animal Rights and Anti-Nuclear Protests", Social Problems, 42-4, 1995, pp. 493-512.

69. Traïni C., « L’Opposition à la tauromachie », in Traïni C. (ed.), op.cit., pp. 193-213. 
que les images, mêmes les plus répulsives, ne sauraient expliquer à elles seules l'engagement. En l'occurrence, l'analyse iconographique doit toujours être menée conjointement à une interrogation sur les « dispositions à l'indignation » des individus et sur les conditions de transformation de ces dispositions en pratique. Cela dit, on ne saurait sous-estimer ce que peut « faire » l'image à ses récepteurs, comme l'illustre encore cet extrait tiré d'un entretien avec Cleews Vellay, le futur président d'Act Up :

«En 1989, il y avait une dizaine de mecs qui étaient là avec des Tshirts noirs "SILENCE = MORT", "SILENCE = DEATH", avec le triangle rose. C’est là que j’ai vu pour la première fois Act Up [...]. Quand j'ai vu ces T-shirts, y’a eu une espèce de déclic en moi. C'est vraiment ça qu'il fallait faire, c'était là qu'était ma place $[\ldots]^{71}$.»

L'image occupe une place centrale dans les mobilisations collectives. Pourtant, elle reste encore peu étudiée par les sociologues et rencontre même, au niveau le plus basique, toutes les difficultés à être considérée comme un « instrument de collecte des informations 72 ». L'ambition de cet article était précisément de revenir sur les raisons de cette indifférence, d'essayer de montrer en quoi l'image peut être investie comme objet d'étude légitime de la sociologie des mouvements sociaux et d'esquisser quelques pistes de recherche possibles. On espère ainsi avoir convaincu de l'intérêt de " passer à l'image 73 » pour étudier les phénomènes protestataires - intérêt qu'illustrent les contributions réunies dans le cadre de ce numéro collectif. À vrai dire, jamais les conditions ne semblent avoir été aussi favorables à une intégration des images comme matériau de travail. L'exploitation des sources iconiques apparaît en effet en mesure d'appuyer le développement des tendances paradigmatiques les plus récentes de la recherche. Les images se sont par ailleurs trouvées au cœur de la plupart des mouvements contestataires récents, des révolutions arabes jusqu'aux happenings des Femen. Bien plus, on peut faire le pari que leur utilisation permettrait de relancer "l'imagination sociologique ». Les « crampes mentales» ne sont certes pas faciles à dissiper. Mais plus de deux décennies après les travaux pionniers en France de Pierre Favre, il semble temps de donner enfin une véritable impulsion aux approches iconographiques de l'action collective.

70. Brodiez A., «Emmaüs et le secours populaire français », in Traïni C., op.cit., pp. 79-95.

71. Cité par Christophe Broqua in Broqua C., Agir..., op.cit., p. 70.

72. La Rocca F., "Introduction à la sociologie visuelle », op.cit., p. 39.

73. Pour reprendre l'expression de Laure De Verdalle et Liora Israël in De Verdalle L., Israël L., «Image(s) des sciences sociales (avant-propos) », Terrains E travaux, 2002, 3, p. 9. 